\title{
SOME THEOREMS ON THE CONNECTION BETWEEN IDEALS AND GROUP OF A GALOIS FIELD*
}

\author{
BY \\ OYSTEIN ORE
}

Let $K$ be a Galois field of order $N$ and $G$ the corresponding finite group. We shall in this paper study some connections between the ideals in $K$ and the constitution of the group $G$.

Let $\mathscr{A}$ be an arbitrary ideal in $K$. We then introduce a subgroup $G_{\boldsymbol{A}} \dagger$ of $G$, which we call the group of the ideal $\mathscr{N}$ and which is defined in the following way. The group $G_{A}$ consists of all substitutions $S$ in $G$ having the property that for every number $\alpha$ in $\mathfrak{A}$ the conjugate number $\alpha^{\prime}=S: \alpha$ is also a number in $\mathscr{P}$.

When $\mathscr{A}=\mathfrak{B}$ is a prime ideal the group $G_{P}$ of $\mathfrak{B}$ is equal to the "Zerlegungs" group of $\mathfrak{P}$, studied by Dedekind $\ddagger$ and Hilbert. $\S$ It is known that the group $G_{P}$ is a metacyclic group, and that the form of the prime-ideal decomposition of the corresponding rational prime $p$ is closely connected with the properties of this group.

In $\$ 1$ we study the properties of the general groups $G_{A}$ and completely determine their construction. In $\S 2$ we deal with the properties of the field $K_{A}$ corresponding to the group $G_{A}$, and we prove that $K_{A}$ is the subfield of $K$ of lowest degree for which there exists an exponent $n$, such that $\mathscr{A}^{n}$ is an ideal in the field. In $\$ 3$ we study another generalization of the "Zerlegungs" field of Hilbert, namely the fields wherein all numbers are congruent to a rational number $\left(\bmod \mathfrak{A}^{\alpha}\right)$ for an arbitrarily great $\alpha$, and for a given $\mathfrak{A}$ we determine all the fields having this property.

In another paper I shall use these results in a more elaborate study of the arithmetic of Galois fields and their subfields.

1. Determination of the group of an ideal. Let $G_{A}$ be the group of the ideal $\mathfrak{A}$; then $G_{A}$ consists of all substitutions in $G$ transforming every number $\alpha$ in $\mathscr{A}$ into another number $\alpha^{\prime}=S: \alpha$ also contained in $\mathfrak{A}$. These substitutions obviously form a group, because the product $S S^{\prime}$ of two substitutions in $G_{\Delta}$

* Presented to the Society, October 29, 1927; received by the editors in October, 1927.

$\dagger$ For typographical reasons, italic letters have been substituted, in subscripts and superscripts, for the corresponding German letters.

$\ddagger$ R. Dedekind, Zur Theorie der Ideale, Göttinger Nachrichten, 1894.

D. Hilbert, Grundzüge einer Theorie des Galoisschen Zahlkörpers, Göttinger Nachrichten, 1894. 
must also change $\alpha$ into $\alpha^{\prime \prime}=S S^{\prime}: \alpha$, where $\alpha^{\prime \prime}$ is also contained in $\mathscr{A}$.

When $S$ is a substitution in $G_{A}$, then $S$ cannot transform a number $\beta$ not contained in $\mathfrak{A}$ into a number $\alpha^{\prime}$ in $\mathfrak{A}$, because from $S: \beta=\alpha^{\prime}$ it follows that the substitution $S^{-1}$ in $G_{A}$ must transform $\alpha^{\prime}$ into $\beta$, which is evidently not possible.

When $\mathfrak{A}$ is an ideal in any subfield $k$ of $K$, the group $G_{A}$ obviously contains the corresponding subgroup $G^{(k)}$ of $G$. On the other hand, there exist ideals $\mathfrak{A}$ not contained in $k$, such that $\mathfrak{A}$ is invariant under all substitutions of the group $G^{(k)}$. In $\$ 2$ we shall, for a given subfield $k$, determine all ideals in $K$ having this property.

In particular, when $[a]=\mathfrak{A}$ is a principal ideal and $a$ a rational integer, then $G_{A}$ is equal to the complete group $G$. From this remark we can derive a more available criterion for the determination of the substitutions of the group $G_{A}$.

The ideal $\mathfrak{A}$ always contains rational numbers, for instance the norm $N=N(\mathfrak{A})$ of the ideal. We can further find a number $\alpha$ in $\mathfrak{A}$ such that the ideals

$$
[N],[\alpha] / \mathfrak{A}
$$

are relatively prime; the principal ideals $[N]$ and $[\alpha]$ consequently have the greatest common ideal factor $\mathfrak{A}$. Let us call $\alpha$ a primary number in $\mathfrak{A}$. When therefore $\beta$ is an arbitrary number in $\mathscr{A}$, a number $\gamma$ can always be determined such that

$$
\beta \equiv \alpha \gamma \quad(\bmod N)
$$

Consequently if a substitution $S$ transforms $\alpha$ into $\alpha^{\prime}$, where $\alpha^{\prime}$ also belongs to $\mathfrak{A}$, the substitution $S$ must be a substitution in $G_{A}$, because the arbitrary number $\beta$ in $\mathscr{A}$ is transformed into the number

$$
\beta^{\prime}=S: \beta \equiv \alpha^{\prime} \gamma^{\prime}
$$

$(\bmod N)$

where $\beta^{\prime}$ must be a number in $\mathfrak{A}$.

THEOREM 1. If $\alpha$ is a primary number in an ideal $\mathfrak{A}$, and $S$ a substitution transforming $\alpha$ into $\alpha^{\prime}$ also belonging to $\mathfrak{A}$, then $S$ is a substitution of the group $G_{1}$ of $\mathfrak{A}$.

The group $G_{A}$ consists of the substitutions of $G$ for which the ideal $\mathfrak{A}$ is invariant. If $r_{\Delta}$ is the order and $s_{A}=N / r_{A}$ is the index of the group $G_{\boldsymbol{A}}$, we can write the whole group $G$ as a partition of co-sets corresponding to $G_{A}$,

$$
G=G_{A}+G_{A} V_{2}+\cdots+G_{A} V_{\bullet_{A}},
$$


where $V_{i}$ are certain substitutions in $G$. Correspondingly we have $s_{\boldsymbol{A}}$ different conjugate ideals

$$
\mathfrak{A}=\mathfrak{A}_{1}, \mathfrak{H}_{2}, \cdots, \mathfrak{A}_{\boldsymbol{\Delta}_{\mathbf{A}}},
$$

where the group of an ideal $\mathfrak{B}_{i}$ is

$$
G_{A_{i}}=V_{i}^{-1} G_{A} V_{i}
$$

For the norm of $\mathfrak{A}$ in $K$ we therefore have the expression

$$
N(\mathfrak{Q})=\left(\mathfrak{A}_{1} \cdots \mathfrak{A}_{\mathbf{P}_{\mathbf{A}}}\right)^{\boldsymbol{r}_{\mathbf{A}}} .
$$

We shall now examine the construction of the groups $G_{A}$ and we first mention the following, almost obvious theorem:

TheOREM 2. The group $G_{A^{a}}$ of a power is equal to the group $G_{A}$ of $\mathscr{A}$.

We also easily prove

Theorem 3. When the norms $N(\mathfrak{A})$ and $N(\mathfrak{B})$ of two ideals $\mathscr{A}$ and $\mathfrak{B}$ are relatively prime, then the group $G_{C}$ of the product $\mathfrak{E}=\mathscr{2} \mathscr{B}$ is equal to the greatest common subgroup $\left(G_{A}, G_{B}\right)$ of the groups $G_{A}$ and $G_{B}$.

When namely $S$ is a substitution in $\left(G_{A}, G_{B}\right)$, then evidently $S$ belongs to $G_{c}$. Conversely, if $S$ is a substitution in $G_{C}$, then $\mathscr{A}$ and $\mathfrak{B}$ must both be invariant with respect to $S$, because if

$$
S: \mathfrak{A}=\mathfrak{A}^{\prime}, S: \mathfrak{B}=\mathfrak{B}^{\prime},
$$

we have from the definition of $G_{C}$

$$
\mathfrak{A}^{\prime} \mathfrak{B}^{\prime}=\mathfrak{H} \mathfrak{B} .
$$

Now the norms $N(\mathfrak{A})$ and $N(\mathfrak{B})$ are relatively prime, so that $\mathfrak{B}$ cannot have any common factor with any of the conjugates $\mathfrak{H}^{\prime}$ of $\mathfrak{A}$, and consequently we obtain from (2)

$$
\mathfrak{A}^{\prime}=\mathfrak{A}, \mathfrak{B}^{\prime}=\mathfrak{B},
$$

and $S$ belongs to $\left(G_{A}, G_{B}\right)$.

Theorem 3 obviously also holds for an arbitrary number of ideals, whose norms are all relatively prime. The problem of the determination of the general group $G_{A}$ is therefore reduced to the case where the norm of $\mathscr{A}$ is the power of a rational prime $p$, and $\mathfrak{A}$ therefore only contains prime ideals dividing $p$.

We now prove

THEOREM 4. If $\mathfrak{A}$ is an ideal containing only prime ideals $\mathfrak{B}$ dividing the same rational prime $p$, we can uniquely write $\mathfrak{A}$ in the form 


$$
\mathscr{A}=\mathscr{A}_{1}^{a_{1}} \ldots \mathscr{A}_{r}{ }^{a_{r}}
$$

where all exponents $a_{i}$ are different, all $\mathfrak{A}_{i}$ relatively prime, and in general

$$
\mathfrak{A}_{\boldsymbol{i}}=\mathfrak{B}_{i} \mathfrak{P}_{\mathbf{i}}^{\prime} \cdots
$$$$
(i=1,2, \cdots, r)
$$

is the product of different, conjugate prime ideals. Then the group $G_{A}$ is equal to the greatest common subgroup of all groups $G_{A_{i}}$.

$$
G_{A_{A}}=\left(G_{A_{1}}, G_{A_{2}}, \cdots, G_{A_{r}}\right) .
$$

It is obvious that a unique representation of $\mathscr{A}$ in the form (3) always exists. If now $S$ is a substitution contained in $G^{\prime}$, where

$$
G^{\prime}=\left(G_{\alpha_{1}}, \cdots, G_{\alpha_{r}}\right), \alpha_{i}=\mathfrak{A}_{i}^{a_{i}^{i}, *}
$$

is the greatest common subgroup of all the groups $G_{\alpha_{i}}$, then $\mathfrak{A}$ is evidently invariant with respect to $S$, so that $S$ is contained in $G_{A}{ }^{*}$ When on the other hand $S$ is a substitution in $G_{A}$, we can prove that $S$ leaves all ideals $\mathscr{U}_{i}{ }^{a}$, unaltered, and consequently belongs to $G^{\prime}$. For if $S: \mathfrak{A}=\mathfrak{A}^{\prime}$ is the transformed ideal of $\mathfrak{A}$, we can only have $\mathfrak{A}=\mathfrak{Y}^{\prime}$ if at the same time $S: \mathfrak{A}_{i}{ }^{a_{i}}=\mathfrak{H}_{i}{ }^{a_{i}}$ for all $i$, because if $S$ transforms a prime ideal $\mathfrak{B}_{1}$ in $\mathfrak{Q}_{1}$ into, for instance, the prime ideal $\mathfrak{P}_{2}$ in $\mathfrak{A}_{2}$, then the ideal $\mathfrak{U}^{\prime}$ must contain the prime ideal $\mathfrak{P}_{2}$ to the power $\mathfrak{P}_{2}{ }^{a_{1}}$ and consequently $\mathfrak{A} \neq \mathfrak{P}^{\prime}$, contrary to the definition of $S$. We therefore have $G_{A}=G^{\prime}$ and if we apply Theorem 2 to the expression (5) for $G^{\prime}$ we obtain the formula (4).

It now only remains to study the groups of ideals having the form

$$
\mathfrak{A}=\mathfrak{B}_{s_{1}} \mathfrak{P}_{s_{2}} \cdots \mathfrak{P}_{s_{t}},
$$

where the prime ideals $\mathfrak{P}_{\text {s }}$ are all different, but factors of the same rational prime $p$.

If $G_{P}$ is the group of an arbitrary prime ideal dividing $p$ (the "Zerlegungs" group of $\mathfrak{B})$, we obtain from (1)

$$
p^{f}=\left(\mathfrak{P}_{1} \cdots \mathfrak{B}_{s p}\right)^{{ }^{P} P}
$$

and from this relation we immediately derive the well known result

$$
p=\left(\mathfrak{P}_{1} \cdots \mathfrak{P}_{{ }_{\mathrm{P}}}\right)^{\bullet}, e=\frac{r_{P}}{f},
$$

where $e$ and $f$ denote the order and the degree of the prime ideal $\mathfrak{B}=\mathfrak{B}_{1}$.

The corresponding groups of the prime ideals in (7),

$$
G_{P_{1}}, G_{P_{2}}, \cdots, G_{P_{x}},
$$$$
\pi=s_{P},
$$

are all conjugate: their greatest common subgroup $H$ is a self-conjugate

* See second foot note on page 610 . 
subgroup of the complete group $G$. We write the group $G$ in co-sets corresponding to $H$ :

$$
G=H+H V_{2}+\cdots+H V_{\iota_{H}},
$$

where $s_{B}$ is the index of $H$, and

$$
V_{2}, \cdots, V_{{ }_{H}}
$$

are certain substitutions in $G$. By means of (8) we construct the factor group $G / H$ of order $s_{H}$. The substitutions of $B$ produce no permutation in the order of the prime ideals in (7),

$$
\mathfrak{P}_{1}, \mathfrak{P}_{2}, \cdots, \mathfrak{B}_{\mathbf{s}_{p}},
$$

but all the substitutions of an arbitrary co-set $H V_{k}$ produce the same permutation

$$
\mathfrak{P}_{c_{1}}, \mathfrak{P}_{c_{2}}, \cdots, \mathfrak{P}_{c_{z}}, \quad \pi=s_{P},
$$

among these ideals. All substitutions

$$
C=\left(\begin{array}{c}
1,2, \cdots, s_{P} \\
c_{1}, c_{2}, \cdots, c_{s_{P}}
\end{array}\right)
$$

thus obtained form a group simply isomorphic with the factor group, so we can suppose the factor group given in the form of this substitution group. It is well known that to every subgroup of the factor group corresponds uniquely a subgroup of $G$ containing $H$.

By means of the factor group $G / H$ we can easily determine the group $G_{A}$ of an ideal $\mathscr{Q}$ having the form (6). The group $B$ is evidently a subgroup of $G_{\Delta}$. When, however, $S$ is a substitution belonging to one of the co-sets $H V_{k}$ in (8), this substitution produces the permutation $C$ (9) among the prime ideals in (7). When therefore the ideal $\mathscr{A}$ is to be invariant with respect to $S$, the substitution (9) corresponding to the co-set $B V_{k}$ must have the form

$$
C=C_{1} C_{2},
$$

where $C_{1}$ represents an intransitive substitution containing only the indices $s_{1}, \cdots, s_{t}$ and similarly $C_{2}$ a substitution containing only the remaining indices. The substitutions in the factor group $G / B$ having the form (8) obviously form an intransitive subgroup of $G / B$ and the corresponding subgroup in $G$ must be the group $G_{\Lambda}$. So we have the following theorem:

THEOREM 5. Let the self-conjugate group $B$ be the greatest common subgroup of all the groups $G_{P_{i}}$ corresponding to the different prime ideals $\Re_{i}$ in $p$, and 
let $G / H$ denote the factor group of $G$ corresponding to $H$. Then the group of an ideal

$$
\mathfrak{A}=\mathfrak{B}_{\iota_{1}} \cdots \mathfrak{B}_{\iota t},
$$

where all factors are different conjugate prime ideals, is the subgroup of $G$ which corresponds to the intransitive subgroup $G_{A}{ }^{\prime}$ of $G / B$ permuting only the indices $s_{1}, \cdots, s_{t}$ inter se and correspondingly permuting the remaining indices mutually.

2. The corresponding fields. To the group $G_{\boldsymbol{A}}$ of an arbitrary ideal $\mathscr{U}$ corresponds uniquely a certain subfield $K_{A}$ of $K$. We call $K_{A}$ the subfield corresponding to $\mathfrak{A}$, and we shall now study the arithmetical properties of this field.

We shall first prove a preliminary theorem concerning an arbitrary subfield $k$ of $K$. Let $G^{(k)}$ be the subgroup of $G$ corresponding to $k$. As we have already remarked in $\$ 1$, all the ideals in $k$ are invariant with respect to the substitutions of $G^{(k)}$, but we can also show the existence of ideals in $K$ not contained in $k$ having the same property, and our first problem is to determine all these ideals.

Let $\mathfrak{p}$ be a prime ideal in $k$ containing a prime ideal $\mathfrak{P}$ in $K$ as a factor. We can then determine the prime ideal decomposition of $p$ in $K$ in the following way: Let $G^{\prime}$ be the greatest common subgroup of $G^{(k)}$ and the group $G_{P}$; then $G^{(k)}$ can be developed in co-sets corresponding to $G^{\prime}$,

$$
G^{(k)}=G^{\prime}+G^{\prime} V_{2}+\cdots+G^{\prime} V_{\bullet},
$$

and it follows easily that $p$ is divisible by $s$ different prime ideals

$$
\mathfrak{P}_{1}=\mathfrak{B}, \mathfrak{P}_{2}, \cdots, \mathfrak{B}_{\text {. }}
$$

We therefore obtain

$$
N_{k}(\mathfrak{B})=\mathfrak{p}^{f_{k}}=\left(\mathfrak{B}_{1} \cdots \mathfrak{B}_{\triangleleft}\right)^{r},
$$

where $N_{k}$ indicates the norm in $k, f_{k}$ is the relative degree of $\mathfrak{B}$, and $r$ the order of the group $G^{\prime}$. Consequently we have the prime ideal decomposition*

$$
\mathfrak{p}=\left(\mathfrak{P}_{1} \cdots \mathfrak{P}_{\mathrm{\imath}}\right)^{\bullet^{\prime}}, \quad e^{\prime}=\frac{r}{f_{k}}
$$

where the exponent $e^{\prime}$ necessarily is a divisor of $N, r$ being the order of a subgroup of $G$.

If now $\mathfrak{A}$ is an ideal in $K$, which is invariant with respect to the group $G^{(k)}$, and we suppose $\mathfrak{A}$ to be divisible by exactly $\mathfrak{P}^{\lambda}$, then $\mathfrak{A}$ obviously must

\footnotetext{
* See, for example, H. Weber, Lehrbuch der Algebra, vol. II, on relative fields.
} 
contain all the prime ideals $\Re_{i}$ dividing $\mathfrak{p}$ in (11) to the same power $\mathfrak{B}_{i}$. Hence we can write

$$
\mathfrak{A}=\left(\mathfrak{P}_{1} \cdots \mathfrak{B}_{\diamond}\right)^{\lambda} \mathfrak{B},
$$

where the ideal $\mathfrak{B}$ is also invariant with respect to $G^{(k)}$, but relatively prime to $\mathfrak{p}$. By reasoning in the same way on $\mathfrak{B}$ we finally conclude that every invariant ideal can be written as a product

$$
\mathfrak{A}=\prod_{\mathfrak{p}}\left(\mathfrak{P}_{1} \cdots \mathfrak{P}_{\bullet}\right)^{\lambda}
$$

corresponding to the different prime ideals $\mathfrak{p}$ in $k$ having common factors with $\mathfrak{A}$. Conversely, if $\mathfrak{A}$ has the form (12), it is evident that the ideal is invariant with respect to $G^{(k)}$.

The necessary and sufficient condition for an invariant ideal can also be expressed in another way. In (11) the exponent $e^{\prime}$ is a divisor of $N$. When we therefore form the $N$ th power of $\mathfrak{A}$, we see from (12) that every term

$$
\left(\mathfrak{P}_{1} \cdots \mathfrak{B}_{8}\right)^{\lambda N}
$$

is equal to a certain power of the corresponding prime ideal $p$ in $k$, so that the ideal $\mathfrak{A}^{N}$ is an ideal in $k$.

THEOREM 6. The necessary and sufficient condition that an ideal $\mathfrak{A}$ be invariant with respect to a group $G^{(k)}$, is that there exist an exponent $n$ such that $\mathfrak{Q}^{n}$ is an ideal in the corresponding subfield $k$.

As a corollary we see that when an ideal $\mathfrak{A}$ is invariant relative to all substitutions of the complete group $G$, then $\mathfrak{\Re}^{N}=[a]$ is a rational principal ideal.*

We remark that when $\mathfrak{A}$ is invariant with respect to the substitutions of the group $G^{(k)}$, then $\mathscr{P}$ is also invariant relative to all subgroups of $G^{(k)}$, and correspondingly it is evident that the ideal $\mathfrak{Q}^{N}$ must be an ideal in all fields having $k$ as a subfield.

Let now $K_{A}$ be the subfield of $K$ corresponding to the group $G_{A}$. Then $\mathscr{Q}$ is evidently an invariant ideal of $G_{A}$ and by Theorem 6 it therefore follows that an exponent $n$ exists such that $\mathscr{Q}^{n}$ is an ideal in $K_{\boldsymbol{A}}$. But by definition $G_{\Delta}$ is the greatest subgroup of $G$ for which $\mathfrak{Q}$ is invariant, and consequently $\boldsymbol{K}_{\mathbf{A}}$ is the field of lowest degree which contains a power of $\mathfrak{A}$.

THEOREM 7 . The field $K_{A}$ corresponding to an arbitrary ideal $\mathfrak{P}$ is the subfield of lowest degree for which there exists an exponent $n$ such that $\mathfrak{P}^{n}$ is an ideal

- D. Hilbert, Über die Zerlegung der Ideale eines Zahlenkörpers in Primideale, Mathematische Annalen, vol. 44 (1894), p. 1. 
contained in the field. Every field that contains a power of $\mathfrak{2}$ contains $K_{A}$ as a subfield.

As an application we suppose that $\mathfrak{A}=\mathfrak{B}$ is a prime ideal, and hence $G_{P}$ is the "Zerlegungs" group and $K_{P}$ the "Zerlegungs" field, introduced by Hilbert. In this case* there exists a prime ideal $\mathfrak{p}_{0}$ in $K_{P}$ of degree $f_{0}=1$ and order $e_{0}=1$ such that

$$
\mathfrak{p}_{0}=\mathfrak{B}^{e}, N_{P}(\mathfrak{B})=\mathfrak{p}_{0}{ }^{f},
$$

where $N_{P}$ indicates the relative norm with respect to $K_{P}$. From Theorem 7 we conclude that $K_{P}$ is the field of lowest degree for which an exponent $n$ exists such that $\mathfrak{P}^{n}$ is an ideal in the field; every other field $k$ having this property contains $K_{P}$ as a subfield. When $n$ is the least exponent for which $\mathfrak{P}^{n}$ is an ideal in $k$, then obviously $\mathfrak{p}=\mathfrak{P}^{n}$ is a prime ideal in $k$. In fields not containing $K_{P}$ as a subfield the prime ideal $\mathfrak{p}$ which is divisible by $\mathfrak{B}$ must also contain some of the conjugates of $\mathfrak{B}$.

ThEOREM 8. The "Zerlegungs" field $K_{P}$ is the field of lowest degree for which ihere exists an exponent $n$ such that $\mathfrak{B}^{n}=\mathfrak{p}$ is a prime ideal in the field. Every other field having this property contains $K_{P}$ as a subfield, and in a field not containing $K_{P}$ the prime ideal $\mathfrak{p}$ divisible by $\mathfrak{B}$ must also be divisible by other prime ideals.

3. The rational congruence-field of an ideal. The "Zerlegungs" field $K_{P}$ has also another very important property. As we have already mentioned, the ideal $p_{0}$ in (13) is of first degree and first order. Hence, if $\omega_{P}$ is an arbitrary number in $K_{P}$, we have

$$
\omega_{P} \equiv a \quad\left(\bmod p_{0}\right),
$$

where $a$ is rational, and, because the prime $p$ contains exactly the first power of $p_{0}$, it follows that a congruence

$$
\omega_{P} \equiv a \quad\left(\bmod p_{0}{ }^{\alpha}\right)
$$

also holds for all $\alpha$, where the rational number $a$ obviously depends on $\alpha$. From (14) and (13) we therefore derive

Theorem 9. When $\omega_{P}$ is an arbitrary number in the "Zerlegungs" field $K_{P}$, then a congruence

$$
\omega_{P} \equiv a
$$

holds for all $\alpha$, where $a$ is a rational number.

* See, for example, D. Hilbert, Bericht uber die Theorie der algebraischen Zahlen, Jahresbericht der Deutschen Mathematiker-Vereinigurig, vol. 4 (1894-95), \$4. 
We say that the field $K_{P}$ is rational $\left(\bmod p_{0}{ }^{\alpha}\right)$, or also $K_{P}$ is a rational congruence-field $\left(\bmod p_{0}^{\alpha}\right)$. As a generalization of Theorem 9 we could propose to determine all subfields $R_{A}$ of $K$, which are rational $\left(\bmod \mathscr{I}^{\alpha}\right.$ ), where $\mathfrak{A}$ is an arbitrary ideal in $K$. We denote by $R_{A}$ the greatest subfield of $K$ having this property and we see that every other rational field (mod $\left.\mathfrak{A}^{\alpha}\right)$ is a subfield of $R_{A}$. We can also suppose obviously that the ideal $\mathfrak{A}$ is the product of different prime ideals in $K$.

Before we examine the rational subfields of $K(\bmod \mathfrak{q})$, we shall give some results on an arbitrary field $k$, which is rational $\left(\bmod \mathfrak{A}_{0}^{\alpha}\right)$, where

$$
\mathfrak{A}_{0}=\mathfrak{p}_{0} \mathfrak{p}_{1} \cdots \mathfrak{p}_{r}
$$

is an ideal in the field. Every number in $k$ then satisfies a congruence

$$
\omega \equiv a
$$

$\left(\bmod \mathfrak{A} \delta^{8}\right)$

and it is obvious that all the prime ideals $\mathfrak{p}_{i}$ in (15) must be of first degree and first order. But we can also prove that $\mathfrak{A}_{0}$ cannot contain two different prime ideals, for instance $\mathfrak{p}_{0}$ and $\mathfrak{p}_{1}$, dividing the same rational prime $p$. Because if we form the equation $f(x)=0$ satisfied by a primitive number $\omega$ in $k$, it follows* that $f(x)$ for all $\alpha$ has a decomposition

$$
f(x) \equiv\left(x-a_{0}\right)\left(x-a_{1}\right) f_{2}(x)
$$

where $f_{2}(x)$ is a rational polynomial. When the discriminant of $\omega_{A}$ is exactly divisible by $p^{\delta}$, we obtain

$$
\omega-a_{0} \equiv 0 \quad\left(\bmod p_{0}^{\alpha-\delta}\right)
$$

and at the same time

$$
\omega-a_{0} \neq 0
$$

$\left(\bmod \mathfrak{p}_{1}^{\delta}\right)$,

so that a congruence (16) cannot hold for all $\alpha$ if $\mathfrak{A}_{0}$ also contains $\mathfrak{p}_{1}$. We have therefore proved

THEOREM 10. If the field $k$ is rational $\left(\bmod \mathfrak{A}_{0}^{\alpha}\right)$, where the ideal $\mathfrak{A}_{0}$ in $k$ has the form (15), then every prime ideal divisor of $\mathfrak{A}_{0}$ is of first degree and first order, and $\mathfrak{A}_{0}$ cannot contain more than one prime ideal dividing the same prime $p$.

A consequence of this theorem is that every field which is rational with respect to all its ideals is necessarily the rational field $R$, because every prime

\footnotetext{
* I refer to my paper Über den Zusammenhang zwischen den definierenden Gleichungen und der Idealtheorie in algebraischen Körpern (erste Mitteilung), Mathematische Annalen, vol. 96 (1926), Chap. II, $\$ 2$.
} 
$p$ must be a prime ideal of the field, and the discriminant is therefore equal to 1 , and by a theorem of Minkowski $R$ is the only field having this property.

When $\mathscr{Q}_{0}$ has the form $(15), k$ must be rational $\left(\bmod p_{i}^{\alpha}\right)$ for $i=1,2, \cdots$, $r$; but conversely we can conclude, that if $k$ is rational $\left(\bmod p_{i}^{\alpha}\right)$ for $i=1$, $2, \cdots, r$, then $k$ must be rational $\left(\bmod \mathfrak{A}_{0}^{\alpha}\right)$. When namely $p_{i}$ divides the rational prime $p_{i}$, the numbers

$$
0,1, \cdots, p_{i}^{\alpha}-1
$$

form a complete system of incongruent numbers $\left(\bmod p_{i}^{\alpha}\right)$, and when we put

$$
M_{i}=\frac{\left(p_{1} \cdots p_{r}\right)^{\alpha}}{p_{i}^{\alpha}}
$$

the rational numbers

$$
\sum_{i=1}^{r} M_{i} a_{i} \quad\left(a_{i}=0,1, \cdots, p_{i}^{\alpha}-1\right)
$$

form a complete system of incongruent numbers $\left(\bmod \mathscr{N}_{0}{ }^{\alpha}\right)$. It therefore follows conversely, that when an ideal $\mathfrak{H}_{0}$ satisfies the conditions of Theorem 10 , then the field $k$ is necessarily rational $\left(\bmod \mathscr{2}_{0}^{\alpha}\right)$.

After these general remarks we return to the problem of the determination of all subfields of $K$ that are rational $(\bmod \mathscr{A} \alpha)$, where $\mathfrak{A}$ is an ideal in $K$. We first suppose that $\mathfrak{A}$ only contains prime ideals dividing a single prime $p$, so that $\mathfrak{A}$ has the form

$$
\mathfrak{A}=\mathfrak{B}_{1} \cdots \mathfrak{B}_{t} .
$$

From Theorem 10 we conclude that in the corresponding field $R_{A}$ there exists a prime ideal $\mathfrak{p}_{0}$ of first order and first degree containing $\mathfrak{A}$. The field $R_{A}$ is obviously a subfield of all the "Zerlegungs" fields of the prime ideals $\mathfrak{B}_{i}$ in $\mathscr{A}$, and consequently is also contained in their greatest common subfield. But $R_{A}$ is generally not equal to this field, as the prime ideals $\mathfrak{P}_{i}$ can be divisors of two different prime ideals of this field.

We shall now determine the field $R_{A}$ by means of the corresponding group $G^{\prime}$. The group $G^{\prime}$ must contain the "Zerlegungs" groups $G_{P_{i}}$ of all divisors $\mathfrak{B}_{i}$ of $\mathfrak{A}$, and conversely we see that every group $G^{\prime}$ containing all $G_{P_{i}}$ corresponds to a field, where the prime ideals $\mathfrak{B}_{i}$ all divide prime ideals of first order and first degree.

When now in particular $G_{P}$ is the "Zerlegungs" group of $\mathfrak{P}=\mathfrak{B}_{1}$ and

$$
V_{2}, V_{3}, \cdots, V_{t}
$$

a system of substitutions in $G$ such that in general $V_{i}$ transforms $\mathfrak{B}$ into $\mathfrak{P}_{i}$, we can prove 
TheOREM 11. The group $G^{\prime}$ of the field $R_{A}$ is the least subgroup of $G$ containing the system

of co-sets in $G$.

$$
G_{P}+G_{P} V_{2}+\cdots+G_{P} V_{\imath}
$$

It is obvious that $G^{\prime}$ contains all the groups $G_{P_{i}}(i=1,2, \cdots, t)$ and in the corresponding field all $\mathfrak{P}_{i}$ must therefore divide prime ideals of first order and first degree, and this prime ideal $p_{0}$ must be the same for all $\mathfrak{B}_{i}$, because if $\mathfrak{p}_{0}$ contains $\mathfrak{B}$ it follows by application of substitutions in $G^{\prime}$ that $\mathfrak{p}_{0}$ is divisible by all $\mathfrak{P}_{i}(i=1,2, \cdots, t)$.

When, more generally, $\mathfrak{A}$ contains prime ideals dividing different primes, we can write

$$
\mathfrak{A}=\mathfrak{A}_{1} \mathfrak{A}_{2} \cdots,
$$

where every factor $\mathfrak{A}_{i}$ has the form (17). From a former remark it then follows that the corresponding rational field $R_{A}\left(\bmod \mathscr{H}^{\alpha}\right)$ is the greatest common subfield of all the fields $R_{A_{i}}(i=1,2, \cdots)$.

4. The inertial group. I also mention finally that in the same way that we generalized the "Zerlegungs" group of Hilbert we can also obtain an inertial group (Trägheitsgruppe) for an arbitrary ideal $\mathscr{A}$. The inertial group $T_{A}$ consists of all substitutions $T$ having the property that

$$
T: \omega \equiv \omega
$$

$(\bmod \mathfrak{2})$

for every number $\omega$ in $K$.

It is obvious that the group $T_{A}$ so defined is a subgroup of $G_{A}$. The construction of the general group $T_{A}$ is simple. We obtain the following from the definition of $T_{A}$ : The inertial group of a composite ideal $\mathfrak{A}$ is equal to the greatest common subgroup of the different prime ideal powers $\Re^{a}$ dividing A.

When $\mathfrak{A}=\mathfrak{B}$ is a prime ideal, the group $T_{P}$ is the "Trägheits" group of Hilbert. When $\mathfrak{A}=\mathfrak{P}^{\mathfrak{a}}, a>1$, Hilbert introduced the notation "Verzweigungs" group (einmal, zweimal etc. überstrichene). It may perhaps be more natural and simpler to use the general conception of the inertial groups and so totally avoid the term "Verzweigungsgruppe."

There are obviously only a finite number of ideals for which $T_{A}$ contains substitutions different from the identical substitution.

Yale UnIVERSTTY, New Haven, Conn. 